\title{
Survey on Evaluation Models of Vertical Handoff Decision Algorithms
}

\author{
Avinash U. Jadhav \\ Department of Computer Engineering \\ Pimpri Chinchwad College of Engineering \\ Pune-44, India
}

\author{
S. S. Sambare \\ Department of Computer Engineering \\ Pimpri Chinchwad College of Engineering \\ Pune-44, India
}

\begin{abstract}
In Heterogeneous systems, Vertical Handoff calculations (VHAs) used to permit mobile terminals (MT) to keep up the system association when versatile hub switch starting with one remote system then onto the next one. The Vertical Handover Decision is NP-Hard issue, thus assessment of all VHA is imperative perspective while working with Vertical Handoff calculations. In this paper, we concentrated on different assessment models which were utilized by past analysts. We likewise concentrated on the different parameters which are imperative for execution examination of VHAs and represent the absence of a legitimate assessment model to look at the different Vertical Handoff Algorithms (VHAs) that have been proposed in the writing.
\end{abstract}

\section{Keywords}

Heterogeneous Networks, Vertical Handoff Algorithms, Mobile Terminals, Evaluation models.

\section{INTRODUCTION}

The next generation wireless networking (4G) is mindful on accomplishing interoperability between diverse system innovations in a consistent way, and on encouraging the client's versatility through a lasting remote association anyplace and whenever [1].

As of now, a percentage of the advanced cells in the business sector are furnished with numerous system interface cards which can join with distinctive remote systems. However this development represents a fascinating test similar to the handoff between different heterogeneous remote systems seamlessly. This critical portability activity is known as the vertical handoff [2].In the last late years, a lot of exploration endeavors [3] have been engaged in this vital and testing versatility process in heterogeneous remote frameworks.

Distinctive Vertical Handoff Algorithms (VHAs) have been proposed in the writing [4]. In any case, there is no any accord on the best way to assess the execution of distinctive VHAs in the examination group. Albeit a few models have been proposed for assessment of the VHAs, this issue has made fascinating difficulties on the grounds that the VHAs have turned out to be more modern and in this manner assessment models must consider an assortment of parameters.

Different late works show enthusiasm for the assessment of VHAs; however some of them don't assess the entire calculation and concentrate just the choice of system innovation stage, which is thought to be the primary segment in the handoff process [7].

In view of the distinctive proposed VHAs, a few studies have concentrated on characterizing which of these arrangements may be ideal for the system choice procedure. Hence, there are studies that utilization distinctive assessment philosophies to achieve this reason.

\section{EVALUATION MODELS}

Many decision algorithms based on multi attributes decision making (MADM) methods have been proposed to deal with the vertical handover algorithm (VHA) problem. The MADM have many methods such as analytic hierarchy process (AHP), simple additive weighting (SAW), multiplicative exponential weighting (MEW), grey relational analysis (GRA), technique for order preference by similarity to ideal solution (TOPSIS), the distance to the ideal alternative (DIA), ELECTRE, VIKOR and WMC (weighted markov chain).

\subsection{Analytic Hierarchy Process}

The vertical handoff decision algorithms considered for comparison needs relative importance of each parameter which is usually given by the set of weights wj. The analytical hierarchical processing (AHP) method is used to determine the weights $[23,24]$ by comparing a pair metrics with the $1-9$ AHP scale. The four traffic classes have different QoS requirements. So, we assigned the different weights according to the importance of parameters in different traffic classes as shown in Table 2.

AHP consists of four steps. One, define the problem and state the goal or objective. Two, define the criteria orfactors that influence the goal. Structure these factors into levels and sublevels. Three, use paired comparisons of each factor with respect to each other that forms a comparison matrix with calculated weights, ranked eigen values and consistency measures. Four, synthesize the ranks of alternatives until the final choice is made.

Individuals and groups use the AHP preference scale in Table 1 to form the comparison matrices.

\subsection{Simple Additive Weighting (SAW)}

In SAW, the overall score of a candidate network is determined by the weighted sum of all the attribute values. The score of each candidate network $\mathrm{i}$ is obtained by adding the normalized contributions from each metric $\mathrm{r}_{\mathrm{ij}}$ multiplied by the importance weight assigned $\mathrm{w}_{\mathrm{j}}$ of metric $\mathrm{j}$. The selected network $\mathrm{A} *_{\mathrm{SAW}}$ is:

$\mathrm{A}_{\text {SAW }}=\operatorname{argmax}_{\mathrm{i} \in \mathrm{M}} \sum_{\mathrm{j}=1}^{\mathrm{N}} \mathrm{wj} \mathrm{rij}$

Where $\mathrm{N}$ is the number of parameters, and $\mathrm{M}$ denotes the number of candidate networks. 
Table 1. Preference Model

\begin{tabular}{|l|l|l|}
\hline $\begin{array}{l}\text { AHP Scale of } \\
\text { Importance for } \\
\text { comparison pair (aij) }\end{array}$ & $\begin{array}{l}\text { Numeric } \\
\text { Rating }\end{array}$ & $\begin{array}{l}\text { Reciprocal } \\
\text { (decimal) }\end{array}$ \\
\hline Extreme Importance & 9 & $1 / 9(0.111)$ \\
\hline $\begin{array}{l}\text { Very strong to } \\
\text { extremely }\end{array}$ & 8 & $1 / 8(0.125)$ \\
\hline Very strong Importance & 7 & $1 / 7(0.143)$ \\
\hline Strongly to very strong & 6 & $1 / 6(0.167)$ \\
\hline Strong Importance & 5 & $1 / 5(0.200)$ \\
\hline Moderately to Strong & 4 & $1 / 4(0.250)$ \\
\hline Moderate Importance & 3 & $1 / 3(0.333)$ \\
\hline Equally to Moderately & 2 & $1 / 2(0.500)$ \\
\hline Equal Importance & 1 & $1(1.000)$ \\
\hline
\end{tabular}

\subsection{Technique for order preference by similarity to ideal situation-TOPSIS}

In Technique for Order Preference by Similarity to Ideal Solution Algorithm (TOPSIS) [4] with M alternatives that are evaluated by $\mathrm{N}$ decision criteria is viewed as a geometric system with $\mathrm{M}$ points in the $\mathrm{N}$ dimensional space. Here, the chosen candidate network is the one which have the shortest distance to the ideal solution and the longest distance to the worst case solution. To compute the network ranking-list, TOPSIS requires the following steps:

Step 1: Construct the normalized decision matrix, which allows comparison across the attributes, this matrix is given by:

$$
r_{i j}=\frac{x_{i j}}{\sqrt{\sum_{i=1}^{m} x_{i j}^{2}}}
$$

Step 2: Construct the weighted normalized decision matrix as $v i j=w j * r i j$.

Step 3: Determine ideal and negative-ideal solutions by:

$A^{+}=\left\{\left(\max _{i \in M} v_{i j} \mid \mathrm{j} \in J\right),\left(\min _{i \in M} v_{i j} \mid \mathrm{j} \in J\right)\right\}$,

$A^{-}=\left\{\left(\min _{i \in M} v_{i j} \mid \mathrm{j} \in J\right),\left(\max v_{i j} \mid \mathrm{j} \in J\right)\right\}$

Where $\mathrm{J}$ is the set of benefit parameters, and $\mathrm{J}$ ' is the set of cost parameters.

Step 4: Calculate the separation measure between the networks and the positive and negative ideal networks by:

$s_{i}^{+}=\sqrt{\sum_{j \in N}\left(v_{i j}-v_{j}^{+}\right)^{2}}, s_{i}^{-}=\sqrt{\sum_{j \in N}\left(v_{i j}-v_{j}^{-}\right)^{2}}$

Step 5: Calculate the relative closeness to the ideal solution

$c_{i}^{*}=\frac{s_{i}^{-}}{\left(s_{i}^{+}+s_{i}^{-}\right)}$

A set of alternatives can now be preference ranked according to the descending order of $\mathrm{c} * \mathrm{i}$. Then the selected network $\mathrm{A} * \mathrm{TOP}$ is:

$A_{\text {Top }}^{*}=\operatorname{argmax}_{i \in M} C_{i}^{*}$

\subsection{Multiplicative exponential weighted MEW}

Using this technique, vertical handoff decision can be expressed as a matrix where each row $\mathrm{i}$ corresponds to the candidate network $\mathrm{i}$ and each column $\mathrm{j}$ corresponds to an attribute (Bandwidth, Delay, etc.). The score Si of network i is

$\mathrm{S}_{\mathrm{t}}=\prod_{\mathrm{j}=1}^{\mathrm{N}} \mathrm{x}_{\mathrm{ij}}^{\mathrm{w}_{\mathrm{j}}}$

Where xij denotes attribute $\mathrm{j}$ of candidate network $\mathrm{i}$, wj denotes the weight of attribute $\mathrm{j}$ and $\sum_{\mathrm{j}=1}^{\mathrm{N}} \mathrm{Wj}=1$

wj is a positive power for benefit metrics ( $\mathrm{xwjij}$ ), and a negative power for cost metrics ( $x-w j i j)$. Since the score is an upper bound, it is convenient to compare each network with the score of the positive ideal network A**. This network is defined as the network with the best values in each metric. (For a benefit metric, the best value is the largest. For a cost metric, the best value is the lowest.) The value of ratio $\mathrm{Ri}$ between network $\mathrm{i}$ and the positive ideal is:

$R_{i}=\frac{\prod_{j-1}^{N} x_{i j}^{w_{j}}}{\prod_{j-1}^{N}\left(x_{i j}^{++}\right)^{w_{j}}}$

The selected network $\mathrm{A} * \mathrm{MEW}$ is obtained as:

$\mathrm{A}_{\mathrm{MEW}}^{*}=\operatorname{argmax}_{\mathrm{i} \in \mathrm{M}} \mathrm{R}_{\mathrm{i}}$

\subsection{Gray Relational Analysis - GRA}

In Grey Relational Analysis (GRA) [6] algorithm, grey relational coefficient (GRC) is used as the coefficient to describe the similarity between each candidate network and the best reference network (an ideal network formed by choosing the best value of each attribute). GRA is usually implemented following three steps: normalization data, defining the ideal sequence, and computing GRC. The normalization of the sequence data is performed according to the three situations (larger-the-better, smaller-the-better, and nominal-the-best) as follows:

$r_{i j}=\frac{x_{i j}-l_{j}}{u_{j}-l_{j}}$,

$r_{i j}=\frac{u_{j}-x_{i j}}{u_{j}-l_{j}}$,

$r_{i j}=1-\frac{\left|x_{i j}-m_{j}\right|}{\max _{u_{j}}-m_{j}, m_{j}-l_{j}}$

Where $u j=\max i \in M x i j, l j=\min i \in M x i j$, and $m j$ is the largest value in the situation of nominal-the-best, for $\mathrm{j}=1,2$, $3, \ldots, \mathrm{N}$. The ideal sequence $\mathrm{x} 0$ is defined to contain the upper bound, lower bound, or moderate bound respectively in larger-the-better, smaller-the-better or nominal-the-better situations. The GRC can be then calculated as following:

$G R C_{i}=\frac{1}{m} \sum_{j=1}^{m} \frac{\Delta_{\min }+\Delta_{\max }}{\Delta_{i}+\Delta_{\max }}$

Where $\Delta_{i}=\left|x_{0 j}-r_{i j}\right|$,

And $\quad \Delta_{\text {max }}=\max _{i \in M, j \in N} \Delta_{i}, \Delta_{\min }=\min _{i \in M, j \in N} \Delta_{i}$

The larger the GRC, the more preferable the network will be. The selected network A* GRA is:

$A_{G R A}^{*}=\arg \max _{i \in M} G R C_{i}$

\subsection{The Distance to the Ideal Alternative DiA}

DIA uses the Manhattan distance to calculate the distance between the attribute values and the positive and negative ideal values of each attribute:

$\mathrm{D}_{\mathrm{j}}^{+}=\sum_{\mathrm{j}=1}^{\mathrm{m}}\left|\mathrm{v}_{\mathrm{ij}}-\mathrm{a}_{\mathrm{i}}^{+}\right|$

$\mathrm{D}_{\mathrm{j}}^{-}=\sum_{\mathrm{j}=1}^{\mathrm{m}}\left|\mathrm{v}_{\mathrm{ij}}-\mathrm{a}_{\mathrm{i}}^{-}\right|$ 
Then, DiA considers the minimum value of $\mathrm{D}+$ and maximum Value of D-.

$\min \mathrm{D}^{+}=\min \mathrm{D}_{\mathrm{j}}^{+}=\min _{\mathrm{j}} \sum_{\mathrm{j}=1}^{\mathrm{m}}\left|\mathrm{v}_{\mathrm{ij}}-\mathrm{a}_{\mathrm{i}}^{+}\right|$

$\max D^{-}=\max D_{j}^{-}=\max _{j} \sum_{j=1}^{m}\left|v_{i j}-a_{i}^{-}\right|$

If we consider the $\left(\mathrm{D}^{+}, \mathrm{D}^{-}\right)$plane, the point (min $\mathrm{Di}+$, max Di-) is defined as the "positive ideal alternative" (PIA).

The best alternative has the shortest distance to the PIA. This absolute distance is calculated as follow.

$\mathrm{R}_{\mathrm{j}}=\sqrt{\left(\mathrm{D}_{\mathrm{j}}^{+}-\min \left(\mathrm{D}^{+}\right)\right)^{2}+\left(\mathrm{D}_{\mathrm{j}}^{-}-\max \left(\mathrm{D}^{-}\right)\right)^{2}}$

The alternative having the smallest $\mathrm{Rj}$ value has the shortest distance to the PIA.

\subsection{ELECTRE}

In Elimination and Choice Translating Priority(ELECTRE) algorithm [7], a reference attribute vector is used to adjust the raw attribute values for the alternative networks before they are compared. The value of each of the attributes in the decision matrix is compared with a corresponding reference attribute value xrefj. An absolute difference between the two values is taken to calculate a new matrix as follows.

$r_{i j}=\left|x_{i j}-x_{j}^{r e f}\right|$

Now in this matrix all attribute values can be considered to have a monotonically decreasing utility. Since a lower value for an adjusted attribute in (1) is considered an indication of a better network in the selection process, each attribute in (1) can be normalized as follows,

$\hat{r}_{i j}=\frac{\max _{i \in M}\left\{r_{i j}\right\}-r_{i j}}{\max _{i \epsilon M}\left\{r_{i j}\right\}-\min _{i \epsilon M}\left\{r_{i j}\right\}}$

Now, is necessary take into consideration the relative importance of each of the attributes involved in the decision about network selection? For the $\mathrm{j}$-th attribute is assigned a weight wj, such that $\sum_{j=1}^{N} \mathrm{Wj}=1$ using the weights, an updatedmatrix is calculated by,

$\overline{r_{i j}}=w_{j} \hat{r}_{i j}$

In order to compare the network alternatives, the concept of concordance and discordance has been introduced in ELECTRE, which are measures of satisfaction and dissatisfaction of the decision maker when one alternative is compared with another. It firstly uses pair-wise comparisons of networks to obtain the concordance set $\operatorname{CSet}(\mathrm{k}, \mathrm{l})$ indicating the attribute of network $\mathrm{k}$ is better than network 1 and the discordance se $\operatorname{tDSet}(\mathrm{k}, \mathrm{l})$ indicating the attribute of network $\mathrm{k}$ is worse than network 1 . The concordance and discordance sets are formed as follows,

$$
\begin{aligned}
& \text { CSet }_{k l}=\left\{j \mid \bar{r}_{k j}>=\bar{r}_{i j}\right\} \\
& \text { DSet }_{k l}=\left\{j \mid \bar{r}_{k j}<\bar{r}_{i j}\right\}
\end{aligned}
$$

Using the concordance and discordance sets, corresponding matrices are constructed. The elements of the concordance matrix $\mathrm{C}$ can be represented as,

$c_{k l}=\sum_{j \in C S e t_{k l}} w_{j}$

The entries for the concordance matrix are not defined for the diagonal. ELECTRE defines the elements of discordance matrix as follows: $d_{k l}=\frac{\sum_{j \in \mathrm{DS} e t}\left|\bar{r}_{k j}-\bar{r}_{i j}\right|}{\sum_{j \in N}\left|\bar{r}_{k j}-\bar{r}_{i j}\right|}$

Similarly, the entries for the discordance matrix are also not defined for the diagonal. A new parameter $\mathrm{Ci}$, called the net concordance index is calculated. $\mathrm{Ci}$ is a measure of dominance of an alternative $\mathrm{i}$ over other alternatives. It can be calculated as follows,

$\hat{C}_{i}=\sum_{j \in N, j \neq i} C_{i j}-\sum_{j \in N, j \neq i} C_{j i}$

Similarly, the term net discordance index $\mathrm{Di}$, is defined as a measure of relative weakness of alternative $i$ over other alternatives and can be calculated as

$\widehat{D}_{i}=\sum_{j \in N, j \neq i} D_{i j}-\sum_{j \in N, j \neq i} D_{j i}$

An alternative with the highest value of net concordance index $\mathrm{C}^{\sim}$ and the lowest value of net discordance index $\mathrm{D}^{\sim}$ would be preferred. However, if it is not the case, the alternatives are ranked based on the concordance and discordance indices and each alternative is ranked by taking the average of these two rankings. The alternative with the highest average ranking is considered to be the best alternative. Alternatives with the same average ranking would be considered equally suited.

\subsection{VIKOR}

For VIKOR [9] method the following steps are required:

Step 1: For each parameter $\mathrm{j}=1,2,3 \ldots \mathrm{N}$, determine the best and the worst values given by:

$F_{j}^{+}=\left\{\left(\max _{i \in M} x_{i j} \mid j \in N_{b}\right),\left(\min _{i \in M} x_{i j} \mid j \in N_{c}\right)\right\}$

$F_{j}^{-}=\left\{\left(\min _{i \in M} x_{i j} \mid j \in N_{b}\right),\left(\max _{i \in M} x_{i j} \mid j \in N_{c}\right)\right\}$

Where $\mathrm{Nb} \subset \mathrm{N}$ is the set of benefit parameters, and $\mathrm{Nc} \subset \mathrm{N}$ is the set of cost parameters.

Step 2: Compute the values of $\mathrm{Si}$ and $\mathrm{Ri}$ for $\mathrm{i}=1,2,3 \ldots \mathrm{M}$ given by:

$s_{i}=\sum_{j \in N} w_{j} \frac{\left(F_{j}^{+}-x_{i j}\right)}{\left(F_{j}^{+}-F_{j}^{-}\right)}$

$R_{i}=\max _{j \in M}\left[w_{j} \frac{\left(F_{j}^{+}-x_{i j}\right)}{\left(F_{j}^{+}-F_{j}^{-}\right)}\right]$

Where wj is the importance weight of parameter $\mathrm{j}$.

Step 3: Compute the values of Qi for $\mathrm{i}=1,2,3 \ldots \mathrm{M}$ given by:

$Q_{i}=\gamma\left(\frac{S_{i}-S^{+}}{S^{-}-S^{+}}\right)+(1-\gamma)\left(\frac{R_{i}-R^{+}}{R^{-}-R^{+}}\right)$,

Where, $S^{+}=\min _{i \in M} S_{i}, S^{+}=\max _{i \in M} S_{i}$,

$R^{+}=\min _{i \epsilon M} R_{i}, R^{-}=\max _{i \in M} R_{i}$,

And parameter $\gamma$ with $0 \leq \gamma \leq 1$ is the weight of the strategy. It also represents the majority of criteria.

Step 4: Given the values for the $\mathrm{Q}, \mathrm{R}$ and $\mathrm{S}$ for all $\mathrm{i} \in \mathrm{M}$, rank the candidate networks in an increasing order. The selected network $\mathrm{A} * \mathrm{~V}$ IK is:

$A_{V I K}^{*}=\arg \min _{i \in M} Q_{i}^{*}$

\subsection{WMC}

The weighted Markov chain (WMC) [8] algorithm includes the following steps:

Step 1: Construction of weighted Markov chain transition matrix MC. Initialize a $\mathrm{M} \times \mathrm{M}$ matrix $\mathrm{MC}=\{$ mcij $\}$ with all 
element values are equal to 0 , in which mcij represents transition probability from alternative pi to the network pj.

Step 2: For each decision factor q, a ranking list is obtained as

$T_{q}=\left[p_{1} \geq p_{2} \geq \cdots \geq p_{M}\right]$

Where " $\geq$ " represents some ordering relation, and $\tau q(p)$ denotes the ranking of alternative $\mathrm{p}$ with regard to factor $\mathrm{q}$.

Step 3: For each mcij in MC, update

$m c_{i j}=m c_{i j}+\frac{w_{q}}{T_{q}\left(p_{i}\right)}$, if $T_{q}\left(p_{i}\right) \geq T_{q}(j)$

Step 4: Computation of stationary probabilities:

$\pi_{j}=\sum_{i=0}^{M} \pi_{i} m c_{i j}, \sum_{j=0}^{M} \pi_{j}=1$

The selected network $\mathrm{A} * \mathrm{WMC}$ is:

$A_{W M C}^{*}=\arg \max _{j \in M}\left(\pi_{j}\right)$

\section{PERFORMANCE OF MODELS}

One of the most important criteria is the total bandwidth and corresponds to WiMAX1, methods as WMC, ELECTRE and VIKOR select this. On the other hand, the available bandwidth is necessary for data transmission, but in the simulation, WLAN2provides a higher available bandwidth than the rest. This causes that methods as SAW, MEW and TOPSIS perform a vertical handoff to WLAN2 to achieve the best connectivity.

On the other hand, GRA algorithm selects WLAN2 for all the vertical handoff decision points.

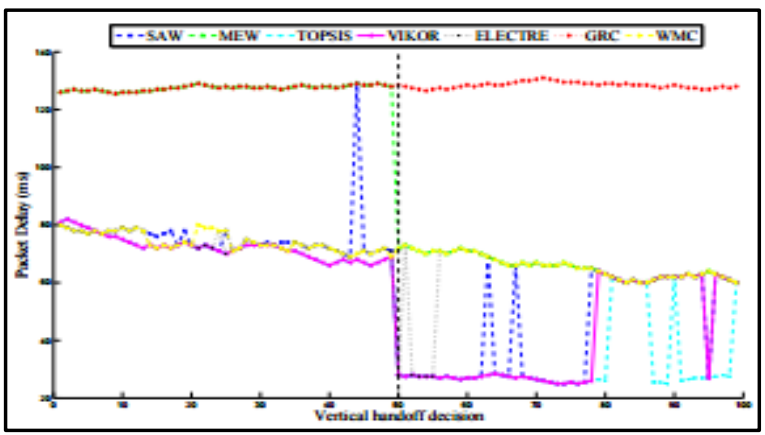

Figure 1: Values of packet delay selected by the decision methods.

Figure 3 shows the available bandwidth achieved by the seven vertical handoff algorithms, decision points 1 to 50 corresponds to case 1 and decision points 51 to 100 to case 3 .

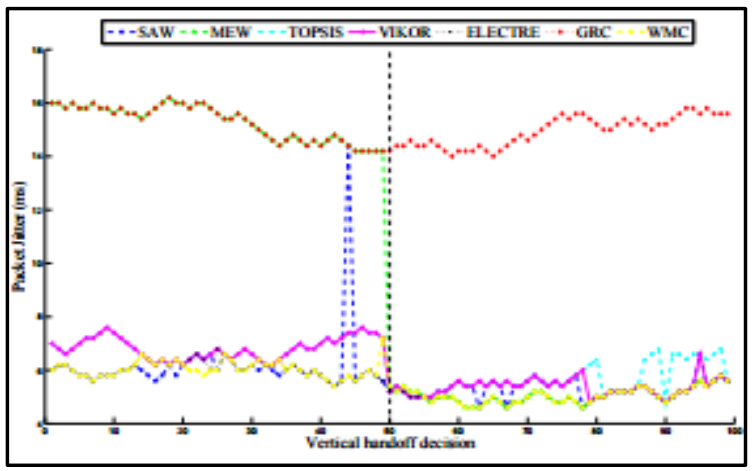

Figure 2: Values of packet jitter selected by the decision methods.
We can see that in case $3 \mathrm{MEW}$ and GRA are able to obtain the highest values of available bandwidth followed by SAW and TOPSIS. On the other hand, VIKOR, ELECTRE and WMC reduce their available bandwidth compared.

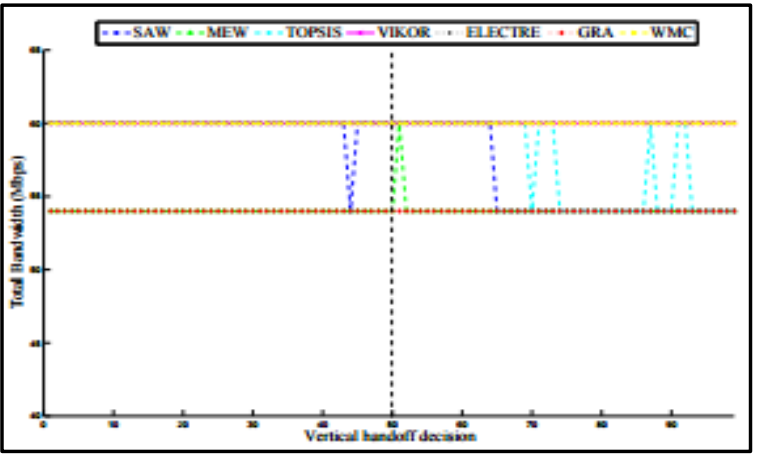

Figure 3: Values of total bandwidth selected by the decision methods

\section{CONCLUSION}

In this paper, we provide a study of several vertical handoff decision algorithms, with the aim of understand its performance for different user applications. Methods as SAW, VIKOR and TOPSIS are suitable for voice connections, these algorithms provide a compromise for achieve the lower values of jitter and delay packet available in a $4 \mathrm{G}$ wireless network. In a data connection case, GRA and MEW algorithms provide the solution with highest available bandwidth necessary for this application.

\section{ACKNOWLEDGMENTS}

I would like to thank my guide Prof S.S.Sambare for his support during my project. Also for their innovative idea who gave me scope for this project work.

\section{REFERENCES}

[1] M. Kassar, B. Kervella, and G. Pujolle, An overview of vertical handover decision strategies in heterogeneous wireless networks, Computer Communications, vol. 31, pp. 2607-2620, 2008.

[2] N. Nasser, A. Hasswa, and H. Hassanein, Handoffs in Fourth Generation Heterogeneous Wireless Networks," IEEE Communications Magazine, vol. 44, no. 10, pp. 93103, October 2005.

[3] K. Yoon and C. Hwang, Multiple Attribute Decision Making: AnIntroduction. S age Publications, 1995.

[4] W. Zhang, "Handover Decision Using Fuzzy MADM in HeterogeneousNetworks," 2004 IEEE Wireless Communications and Networking Conference,Atlanta, USA, March 2004

[5] E. Stevens-Navarro and V. W.S. Wong, Comparison between Vertical Handoff Decision Algorithms for Heterogeneous Wireless Networks,Proc. of IEEE Vehicular Technology Conference, Melbourne, Australia,May 2006.

[6] Q. Song and A. Jamalipour, Network selection in an integrated wirelessLAN and UMTS environment using mathematical modeling andcomputing techniques, IEEE Wireless Communications, pp. 42-48, June2005.

[7] F. Bari and V. Leung, Application of ELECTRE to network selection in aheterogeneous wireless network environment, in Proc. of IEEE WirelessCommunication 
and Networking Conference (WCNC'07), Hong Kong,China, March 2007.

[8] Y. Wang, P. Zhang, Y. Zhou, J. Yuan, F. Liu, and G. Li, HandoverManagement in Enhanced MIH Framework for Heterogeneous WirelessNetworks Environment, Journal of Wireless Personal Communications, vol. 52, no. 3, pp. 615-636, Feb. 2010.

[9] J.R. Gallardo-Medina, U. Pineda-Rico and E. StevensNavarro, VIKOR Method for Vertical Handoff Decision in Beyond 3G Wireless Networks, in Proc. of IEEE International Conference on Electrical Engineering, Computing Science and Automatic Control (CCE'09), Toluca, Mexico,2009.

[10] L. Wang and D. Binet, MADM-based network selection in heterogeneouswireless networks: a simulation study, in Proc. of Wireless VITAE,Aalborg, Denmark, May 2009.

[11] Gowrishankar, G. N. Sekhar, and P. S. Satyanarayana, Analytic performability model of vertical handoff in wireless networks, Journal of computer science, vol. 5, no. 6, pp. 445-450, 2009.

[12] 12. J.S Kim, E Serpedin, DR Shin, K Qaraqe, Handoff triggering and network selection algorithms for loadbalancing handoff in CDMA-WLAN integrated networks. EURASIP

[13] J.Wirel. Commun. Netw 2008, 136939 (2008) (http://jwen, 2008).

[14] eurasipjournals.com/content/2008/1/136939

[15] AEzzouhairi, A Quintero, S Pierre, A fuzzy decision making strategy for vertical handoffs. Canadian Conference on Electrical and Computer Engineering, 2008. CCECE 2008 ((Ontario, Canada, 2008), pp).

[16] Q He, A fuzzy logic based vertical handoff decision algorithm between WWAN and WLAN. 2nd International Conference on Networking and Digital Society (ICNDS), 2010, vol 2 ((Wenzhou, China, 2010), pp).

[17] T Yang, P Rong, A fuzzy logic vertical handoff algorithm with motion trend decision. 6th International Forum on Strategic Technology (IFOST), 2011, vol. 2 ((Harbin, China, 2011), pp).

[18] A Aziz, S Rizvi, N Saad, Fuzzy logic based vertical handover algorithm between LTE and WLAN. International Conference on Intelligent and Advanced Systems (ICIAS), 2010 ((KLCC 2010).

[19] KS Trivedi, S Dharmaraja, X Ma, Analytic modeling of handoffs in wireless cellular networks. Inf. Sci 148(1-4), 155-166 (http://www, 2002).

[20] sciencedirect.com/science/article/pii/S002002550200292 $\mathrm{X}$
[21] GN Sekhar, PS Satyanarayana, Gowrishankar, Analytic performability model of vertical handoff in wireless networks. J. Comput. Sci 5, 1549-3636 (2009).

[22] H Ramesh Babu, G Raju, P Satyanarayana, Gowrishankar, Performability model of vertical handoff in wireless data network. The Fourth International Conference on Wireless and Mobile Communications, 2008. ICWMC'08 ((Athens, Greece, 2008), pp).

[23] 3GPP: IMT -2000 QoS classes TSG 17, 3rd Generation Partnership Project (3GPP) 3GPP: QoS concepts and architecture (TS 23), . 107, 3rd Generation Partnership Project (3GPP) 2009

[24] E Stevens-Navarro, U Pineda-Rico, J Acosta-Elias, Vertical handover in beyond third generation (3G) wireless networks. Int. J. Future Generation Commun. Netw 1, 1-8 (2010)

[25] K Teknomo, Analytic hierarchy process (AHP) tutorial(http://people),revoledu.com/kardi/tutorial/AHP/i ndex.html

[26] TL Saaty, in Fundamentals of Decision Making and Priority Theory with the Analytic Hierarchy Process. The Analytic Hierarchy Process, vol. 6 (RWS Publications, Pitsburgh, 2000)

[27] J Stein, Survey of IEEE802.21 media independent handover services, (http://www), cse.wustl.edu/jain/cse574-06/ftp/handover/index.html webcite

[28] E Stevens-Navarro, Y Lin, V Wong, An MDP-based vertical handoff decision algorithm for heterogeneous wireless networks. IEEE Trans. Veh. Technol 57(2), 1243-1254 (2008) (Performance Engineering Lab, Derry, Northern Ireland, 2007)

[29] J Mendel, Fuzzy logic systems for engineering: a tutorial. Proc. IEEE 83(3), 345-377 (1995).

[30] S Rajasekaran, G Pai, in Neural Networks, Fuzzy Logic and Genetic Algorithms: Synthesis and Applications ((Prentice-Hall of India, 2004), http://books), google.co.in/books?id=HDg7wCP_bmUC

[31] A Celikyilmaz, I Türkşen, in Modeling Uncertainty with Fuzzy Logic: With Recent Theory and Applications. Studies in Fuzziness and Soft Computing ((Springer, 2009), http://books), google.co.in/books?id=XNHzqfvMTbwC

[32] G Bolch, in Queueing Networks and Markov Chains: Modeling and Performance Evaluation with Computer Science Applications ((Wiley-Interscience Publication, Wiley-Interscience, 2006), http://books),.google.co.in/books?id=8Mei8w6YUHYC 